\title{
Research on Application of Tennis Wall in Tennis Teaching
}

\author{
Bai Yunfan \\ Labor Union of XinYu University, Xinyu, Jiangxi, China, 338000 \\ 346591653@163.com
}

Keywords: Tennis wall, Tennis teaching, Training methods

\begin{abstract}
The tennis wall can solve some problems in university tennis teaching, such as the tennis locations cannot meet the student populations. However, the efficiency of the tennis teaching based on the tennis wall is unknown. This research firstly gives the concept, advantages and disadvantages of the tennis wall, then expounds the methods of the application of tennis wall in tennis teaching, and finally draws the conclusion that tennis wall plays an obvious role in improving students' tennis skill in order to provide some references for the tennis teachers in universities.
\end{abstract}

\section{Introduction}

Tennis was introduced into China with the spreading of western modern sports in the nineteenth century. The development of the sport in China has a history of 100 years. But in the past, the tennis has been regarded as a noble sport. After the reform and opening up, as China's economic development and the improvement of people's living standard, tennis is becoming increasingly popular. It has become a popular sport loved by the people. The tennis movement with its own unique charm has attracted many people to participate in this sport. The country set off a wave of unprecedented heat of tennis. Universities are no exception. More and more students learning tennis, however, many universities cannot open tennis class due to inadequate locations. Many universities also built many tennis courts, the introduction of professional tennis teachers, but because of the increase too fast in students' tennis learning, many school tennis courts far cannot meet the teaching needs, more do not talk to go up to meet the needs of students in extracurricular physical exercise. Tennis is one of higher technical requirements of the project, students in this case it is very difficult to learn basic skills. The author carried a semester teaching experiment using the tennis wall for college tennis elective course. It proved that the reasonable use of tennis wall can solve the college tennis elective teaching problems such as too many students, too little space and too short time. This paper can provide some references for improving the tennis teaching efficiency.

\section{Concept, Advantages and Disadvantages of Tennis Wall}

\section{A: Concept of Tennis Wall}

Tennis wall refers to the wall which has a specific height, width, hardness and has the marks or patterns in accordance with the requirements for tennis training. The origin of the tennis wall can be traced back to 1850. According to the relevant records, a school named Harrow School built a few walls so that the students can play tennis against the wall as tennis is relatively less in In England. This is also the tennis wall appeared for the first time in history. To build a suitable for the training of tennis wall, must fully consider the purpose and method of using it. We know that the current is just tennis wall of the tennis court, assisted the main function of it is tennis training exercises. These tennis wall requirements should be from the tennis venue, space and technology requirements are 
considered. The tennis wall height, Public opinions are divergent. The site construction needs considering the cost, economic and practical requirements. As the height requirement of the tennis court blocking net cannot be less than 3.05 meters in the national standards, the tennis wall height requirements should also be not less than 3.05 meters.

\section{B: Advantages of Tennis Wall}

Tennis wall exercises can increase exercise density and improve the teaching efficiency. As the distance of the wall is only half of the actual site, like the opponent has been standing in the net, the rhythm quickly doubled. Kinematic density is also great. A tennis wall can be dozens of people at the same time exercise, unit time increased the number of practitioners, increase the density of practice teaching in the classroom, which improve the efficiency of classroom teaching. This not only requires you to act quickly. People of different levels, hit the wall feeling and harvest are not the same. Tennis wall ball landing is relatively stable, easy to master the technical movements. Strength of wall practice, practitioners can independently control practice by proper strength practice, practice the ball back strength for, reduce practitioners the ball back to the difficulty, is conducive to improve the practice skill mastery and self-confidence. Practice using the tennis wall, unlimited time, human factors, very convenient. Practitioners can arrange exercises according to their own time. The contents and methods of practice can be independently arranged according to their level and demand.

\section{C: Disadvantages of Tennis Wall}

As the distance is only half of the actual site, the returning ball is very quick. It is difficult to receive the ball, which can easily lead to the bad habits of beginners such as pulling the shoot too fully. This point is very dangerous and the action has not been established for beginners, because each of the walls once is an enhanced memory. Also hitting the wall back the ball with the site to return the ball is different, sense of distance shots, movement, rhythm and intensity is also a wall is not provided by the, the beginners who want to hit the wall skilled out again to rally, the feeling is completely different. Foundation is not good to hit the wall, always hit the ball fly, very easy because often pick up the ball and frustrated, and affect the enthusiasm of tennis. Because the ball hit of the wall is easy to judge, it will reduce the ability to predict. And hitting the wall on the placement of the judge with the stadium on the anticipation is completely different, not much help to improve the ability to predict. A player practice with a speeches wall is boring. The actually fierce practice with other players will be much more interesting. Moreover, the stroking force of the tennis wall is too large and the rebound force of the wall may hurt the practitioners.

\section{Application of Tennis Wall in Tennis Teaching}

\section{A: Single-player Practice}

Facing the wall tennis ball wall, the practitioners can stand in the 1-2 meters distance tennis wall, with medium strength using the military throwing. The ball to the wall about 1.5 meters of mark points on ball ran into a wall after landing first with both hands to pick up, skilled hands after try with one hand, repeated practice, until skilled so far. Forehand and backhand of tennis technology is the most basic and common technique. The forehand and backhand technique is the foundation, so it is the key technology to learn tennis. In practice, let the players stand in front of tennis wall 9 meters, were thrown from the practice ever since. The hitting target is in front of the wall as a symbol of 1.5 meters. The first place point is a hit once. The ball rebounded after the hand to catch the ball repeatedly. The practitioner should experience the hitting action essentials in the stage. The high quality requirements are not very essential. After the practice of a preliminary grasp of positive and backhand action essentials, each action should be continuous practice. This technology can give 
their own master, increase the difficulty, and adjust the hitting power and direction stability. When the practitioner masters the backhand, he can practice the game using the tennis wall.

\section{B: Multi-player Practice}

The tennis wall can also support multi-player practice. Similar with the transposition table tennis doubles in the same rhythm, after hitting and to quickly move out of position. Because the two practitioners are close, they should be caution. The absence of coaches and sparring situation can still focus on practice, the basic technology to achieve as soon as possible. Note that the beginning not too much force, force is not well controlled more easily depends on the wrist to return the ball in haste, the wrong action after the formation of it would be very difficult to get rid of. Tennis beginners can pay attention to grasp, hit the wall tennis method also has the skill, and it can directly affect you for playing tennis technology to improve fast and slow. So the master hit the wall to learn tennis, don't ignore the above mentioned action. Tennis teaching, tennis wall practice difficulty is relatively small, for beginners is relatively easy to grasp, but practice content and relatively dry, exercise prone to mental fatigue and boredom, is not conducive to the development of tennis teaching. So in the tennis teaching practice on the wall, we can take double and multi person continuous batting practice to increase the interest of the practice and enhance the practice enthusiasm of practitioners.

\section{Experimental Design and Conclusion}

\section{A: Experimental Design}

In order to verify the effect of the tennis wall in the tennis teaching, two classes' students in Xinyu University were selected as the research object to carry out the experimental study. We used two group pretest and posttest experimental design. Experiment time: September 2015 - January 2016, location: Xinyu University tennis court, total hours: 60 hours.

The first step: in two classes in each class were randomly selected 15 people, divided into two groups, each group of 15 people. Before the experiment, two groups of basic level tests. Respectively, the experimental group and control group "form" and "function", "health" and "technology" test. This way can ensure the fairness and reduce the packet before the experiment on the experimental results. Second step: in the experimental group and the control group continued to use the test of unified test method. The racket to play all kinds of landing the ball as far as possible repeatedly no mistakes is to cultivate the feeling of the ball racket. On the wall are backhand exercises. We can hit a ball to keep the action stability, continuously than a few rounds. Tennis elective class to wash because the ball hit the wall after the rebound quickly is often too late to hit the ball, in order to effectively return the ball only as soon as possible the racket. Put the ball close to the wall to wall, beating continuously before landing as much as possible to ensure continuous hitting times.

\section{B: Experimental Results}

All of the participants were tested in the terms of students' body shape, physical quality and technical level and be recorded in indicators form. Through the experiment of tennis wall training and non-training of tennis wall students to keep track of, before and after the experiment, respectively, measured in specific regions of the backhand 10 times each batting average success rate and two people inside of pullback of composite number as the test index, explore tennis wall on teaching effect influence and evaluation. Height, weight and other indexes are important indexes to reflect the level of physical development and to master the student body symmetry. The growth and nutritional status has important significance and physiological function of student data control at the same time, the physical quality and the movement ability has a significant impact. Two groups of 
test data as control statistical evaluation index, before the formal trial by corresponding to the two groups of students index test, draw the following conclusion: two groups of students "physical form" index $\mathrm{p}>0.05$, indicating that the two groups of data, no significant difference. As the two groups of students' physical quality index $\mathrm{p}>0.05$, indicating that there is no significant difference between the two groups of data.

\section{C: Conclusions and Suggestions}

This paper draws the following conclusions: Tennis teaching wall training to master has a positive role in every stage of the technology is reasonable choice according to their own master tennis technology training. The experimental group and control group of experimental index, show the significant difference in the before and after the experiment. The results indicate that the tennis wall training has the positive effect on the students' tennis technical mastery. Tennis wall training in tennis teaching is a good way to test and improve the technology, to discover and correct the mistakes in time, and to avoid the wrong actions.

Recommends are as follows: We Summarize the previous research results, that our country tennis movement rapid development momentum in recent years, particularly prominent performance. The number of participants and tennis facilities continue to increase, improving the practical value of the tennis wall. However the number is not high and has not caused enough attention. Using the tennis wall fully and appropriately can effectively alleviate the adverse effects caused by the lack of space and help students to master the technology quickly. Tennis teaching wall training is a good way of self-inspection and improve, can timely discover and correct action, action shape. We should pay attention to the actual effect and avoid the bad effects of the tennis wall. In addition, tennis wall training of tennis teaching effect is positive, but in order to better teaching and training, the tennis wall training must be combined with the regular training venues in order to make students better grasp the tennis technology.

\section{References}

[1] Li Xiaokun, Zhao Xitang, Wall Tennis: Method and Significance, J., Journal of Zhaoqing University, 2015, 36(2): 71-76.

[2] Ma Dahui, The Study on Function of Tennis Wall in University Tennis Elective Course, J., Journal of Jilin Institute of Physical Education, 2010, 26(1): 104-105.

[3] Liu Jingchuan, Wall of Tennis Training Method in Tennis Teaching Research, D., Shandong Institute of P.E. and Sports, 2014.

[4] Yu Nande, The Evaluation of Tennis Teaching Results with Tennis Wall's Constructing, D., Xi'an Physical Education University, 2012. 